\title{
An Autonomous Decentralized Supply Chain Planning and Scheduling System
}

\author{
Tatsushi Nishi
}

\section{Introduction}

For manufacturing industries, the integration of business processes from customer-order management to delivery (Supply Chain Management) has widely been received much attention from the viewpoints of agile and lean manufacturing. Supply chain planning concerns broad activities ranging network-wide inventory management, forecasting, transportation, distribution planning, production planning and scheduling, and so on (Jeremy, 2001; Simon et al., 2000). Various supply chain models and solution approaches have been extensively studied in previous literature (Vidal \& Goetschalckx, 1997). These models are often divided into the following three categories:

1. Integration of production planning among several companies (Mckay et al., 2001)

2. Integration of production planning of multi-sites in a company (Bok et al., 2000)

3. Integration of production planning and distribution at a site from the pro¿ curement of raw materials, transportation to the distribution of intermediate or final products to the customers (Rupp et al., 2000).

The purpose of this work is to address an autonomous decentralized systems approach for integrated optimization of planning and scheduling for multistage production processes at a site with respect to material requirement plano ning, production scheduling and distribution planning. One conventional apđ proach that has been used for planning and scheduling is a hierarchical de吾 composition scheme (Bitran \& Hax, 1977). Planning concerns decisions about \& the amount of products to be produced over a given time so as to maximize $\&$ the total profit. Scheduling involves decisions relating to the timing and se$\bar{\Phi}$ quencing of operations in the production processes so as to satisfy the producO tion goal that is determined by the planning system. Tan (2001) developed a 
hierarchical supply chain planning approach and a method of performance management.

Since in the hierarchical approach, there is practically no feedback loop from the scheduling system to the planning system, the decision made by the scheduling system does not affect the decision at the planning stage; however, the decision made by the planning system must be treated as a constraint by the scheduling system. Therefore, it becomes difficult to derive a production plan taking the precise schedules into account for the hierarchical systems. It is necessary to integrate the scheduling system and the planning system for global optimization of the supply chain (Wei, 2000).

A simultaneous multi-period planning and scheduling model has been proposed by Birewar \& Grossmann (1990) where the scheduling decisions are incorporated at the planning level. It has been demonstrated that the planning profit is significantly increased when planning and scheduling decisions are optimized simultaneously. The disadvantage of their approach is that the planning and sequencing model is restricted to a certain class of simple problems, because an extremely large number of binary variables are needed to solve integrated planning and scheduling problems. Moreover, it is requested that the models of subsystems comprising an SCM system need to be flexible to deal with the dynamically changing environment in a practical SCM. The integrated large-scale models, however, often become increasingly complex. As a result, it becomes very difficult to execute the new addition of constraints and/or the modifications of the performance criterion so as to cope with unforeseen circumstances.

SCM systems must satisfy new requirements for scalability, adaptability, and extendibility to adapt to various changes. If the decisions taken at each subsystem are made individually while aiming to optimize the entire SCM system, it is easy for each subsystem to modify its own model in response to various requirement changes. Distributed planning and scheduling systems have been proposed as an architecture for next-generation manufacturing systems (NGMS). These architectures are often referred to as multi-agent systems, wherein each agent creates each plan locally within the shop floor and each agent autonomously resolves conflicts among plans of other agents in a distributed environment.

Hasebe et al. (1994) proposed an autonomous decentralized scheduling system that has no supervisory system controlling the entire plant with regard to creating schedules for multi-stage production processes. The system comprises a 
database for the entire plant and some scheduling subsystems belonging to the respective production stages. Each subsystem independently generates a schedule for its own production stage without considering the schedules of the other production stages. However, a schedule obtained by simply combining the schedules of all production stages is impracticable in most cases. Therefore, the scheduling subsystem contacts the subsystems of the other production stages and obtains the schedule information of those stages to generate a new schedule. Schedules are generated at each stage and data are exchanged among the subsystems until a feasible schedule for the entire plant is derived. The effectiveness of the autonomous decentralized scheduling system for flowshop and jobshop problems is discussed by Hasebe et al. (1994).

An autonomous decentralized supply chain optimization system comprising three subsystems: material requirement planning subsystem, scheduling subsystem and distribution planning subsystem has been developed. A nearoptimal plan for the entire supply chain is derived through the repeated optimizing at each subsystem and exchanging data among the subsystems. In Section 2 we briefly review distributed planning and scheduling approaches. Supply chain planning problem is stated in Section 3. The model structure and the optimization algorithm of the autonomous decentralized system are developed in Section 4. In Section 5 we compare the proposed method with a conventional planning method for a multi-stage production process. Section 6 summarizes conclusion and future works.

\section{Distributed planning and scheduling}

There have been several distributed planning and scheduling approaches under the international research program called Intelligent Manufacturing Systems (IMS). For example, the biological-oriented manufacturing system (BMS) is an evolutionary approach that contains DNA-type information and BN-type information acquired at each subsystem (Ohkuma \& Ueda, 1996). For the holonic manufacturing system (HMS), intelligent agents called "holons" have a physical component as well as software for production planning and scheduling. A hierarchical structure is adopted to reduce complexity and to increase modularity (Gou et al., 1998; Fisher, 1999).

These distributed planning and scheduling approaches can be classified into hierarchical, non-heterogeneous, and heterogeneous algorithms according to the structure of the distributed systems (Tharumarajah \& Bemelman, 1997). 
Distributed Asynchronous Scheduling (DAS) is organized by three hierarchical agents: operational, tactical, and strategic agents. The constraints are propagated by the message passing through DAS schedulers (Burke \& Prosser, 1990). The non-heterogeneous structure is used as a combination of distributed agents and the conflict coordinator when the coordination between the subsystems cannot be resolved. Maturana \& Norrie (1997) addressed a mediator architecture where coordination of subsystems is dynamically achieved by employing the virtual systems created as needed for coordination. On the other hand, a heterogeneous structure resolves all conflicts among the subsystems without any other subsystems. Smith (1980) proposed a contract net protocol where each heterogeneous agent negotiates with another by receiving and awarding bids.

The algorithms of distributed planning and scheduling approaches can be classified into non-exhaustive or exhaustive approaches according to the conflict resolution and coordination method. In the non-exhaustive algorithm, the number of attempts at coordination is limited to the number of trials required for obtaining a feasible solution without consuming computational expenses (Shaw, 1987). The exhaustive algorithm is founded on the iterative-search based coordination method for obtaining a near-optimal solution, though the solution may only produce a locally optimal solution. The approach employed in this paper is an exhaustive approach with a heterogeneous structure having no supervisory system. The supply chain planning problem for a single-stage production system can be decomposed into a material requirement planning subproblem, a scheduling subproblem and a distribution planning subproblem following the principle of Lagrangian decomposition and coordination approach based on the mathematical programming method (Nishi et al., 2003). This method has been applied to planning and scheduling methods in many previous studies (Gupta et al., 1999; Gou et al., 1998; Hoitomt et al., 1993).

The autonomous decentralized approach features the characteristic that each subsystem has an optimization function for each subsystem based on the idea of decomposition and coordination. Most of the conventional distributed approaches have a hierarchical structure, where a supervisory system or a coordinator makes a decision by using the information obtained by the subsystem. Even though the decisions are created by each subsystem, it is still necessary to use some protocols for coordination. For conventional systems, it is necessary to reconstruct these protocols when the new constraints or the performance criterion is modified. By adopting the structure of the proposed system, the 
proposed approach has a plenty of flexibility to accommodate various changes such as modification of constraints or performance criteria in each subsystem. In the following section, the supply chain optimization problem is stated. Then, the mathematical formulation of the problems is described.

\section{Supply chain planning problem}

The multi-stage flowshop production process is divided into multiple production stages by taking into account the technical and/or managerial relationships in the plant shown in Figure 1. In this study, we assume that the plant satisfies the following conditions.

1. Total planning period is divided into a set of time periods. For each time period, the lower and the upper bound of the production demand of products are given. If the amount of delivery is lower than the lower bound, some penalty must be paid to the customer.

2. Transportation time and transportation cost from supplier of raw material to the plant, and from the plant to customers are negligible.

3. The lead-time at the supplier of raw material is negligible. However, the ordered raw material arrives at the production process only on a prespecified date.

4. Production site has flowshop production line. Each production stage consists of a single batch machine. The amount of product per batch and the production time depend on the product type of the job, but they are fixed for each product.

5. Changeover costs at each stage depend on the product type of the operation executed successively.

6. The capacity of the storage space for raw materials and final products is restricted. Therefore, the amount of storage of each raw material or final product must be lower than its upper bound. The storage cost is proportional to the amount of stored material and the stored period. 


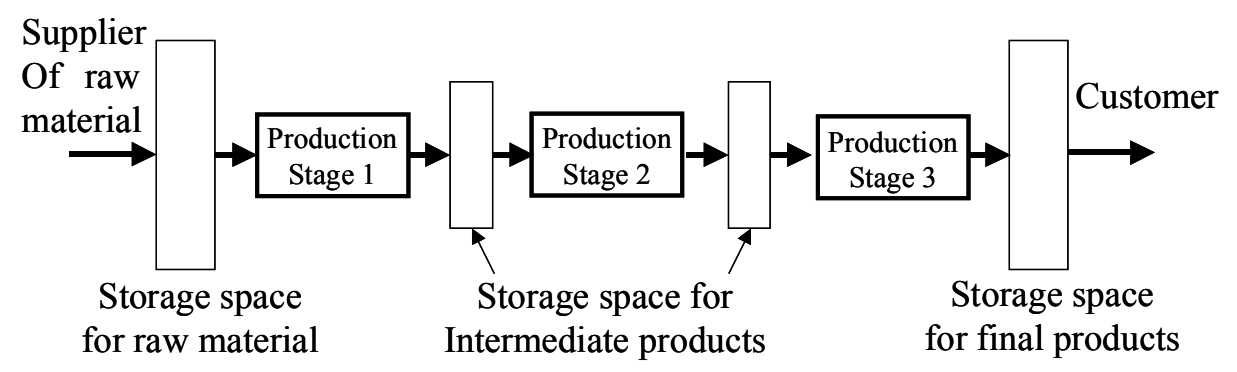

Figure 1. Supply chain for a multi-stage production processes

The supply chain optimization problem for a multi-stage production process is stated as:

The time horizon of planning and scheduling, the lower and upper bound of demand for products, the price of raw materials, inventory holding cost for raw materials, inventory holding cost for final products, the revenue of final product to customer, penalty cost for violating the lower of demand, processing time of operations for each products, changeover cost are given, the problem is to determine the arrival time and the amount of each raw material to storage space for each raw material, the production sequence of operations and their starting times at each production stage, the delivery time and the amount of each product to customers from the storage space for final products to optimize the objective function consisting of material cost, inventory holding cost for raw materials, sequence dependent changeover cost at the production stage, inventory holding cost for final products, production cost, penalty of production shortage.

To solve the above supply chain optimization problem, an autonomous decentralized supply chain optimization system is developed. The details of the proposed system are explained in the following section.

\section{Autonomous decentralized supply chain planning and scheduling system}

Supply chain optimization problems naturally involve the coordination of production, distribution, suppliers of raw material, and customers. Clearly, each of these sections has its own characteristic decision variables and an objective function relating to other sections. To achieve an efficient supply chain 
management, a plan must be developed under the environment which each section is allowed to make independent decisions to its operation so as to optimize its own objective function while satisfying constraints of other sections. (Androulakis \& Reklaitis, 1999). Taking this consideration into account, an autonomous decentralized supply chain optimization system for multi-stage production processes is developed. The supply chain planning problem is decomposed into a material requirement planning subproblem, a scheduling subproblem and a distribution planning subproblem when the material balancing constraints are relaxed following the principle of Lagrangian relaxation method (Nishi et al., 2003). Each subproblem is solved by the subsystem.

\subsection{System structure}

The structure of the system is shown in Figure 2. The total system consists of a database for the entire plant, a material requirement planning subsystem (MRP) and some scheduling subsystems (SS) for respective production stage, and a distribution planning subsystem (DP). The purpose of the MRP subsystem is to decide the material order plan so as to minimize the sum of the material costs and inventory holding costs of raw materials. The SS subsystem determines the production sequence of operations and the starting times of operations so as to minimize the changeover costs and due date penalties. The purpose of the DP subsystem is to decide the delivery plan of each product so as to maximize the profit including inventory costs for final products. The model structure of the decentralized supply chain optimization system is shown in Figure 3.

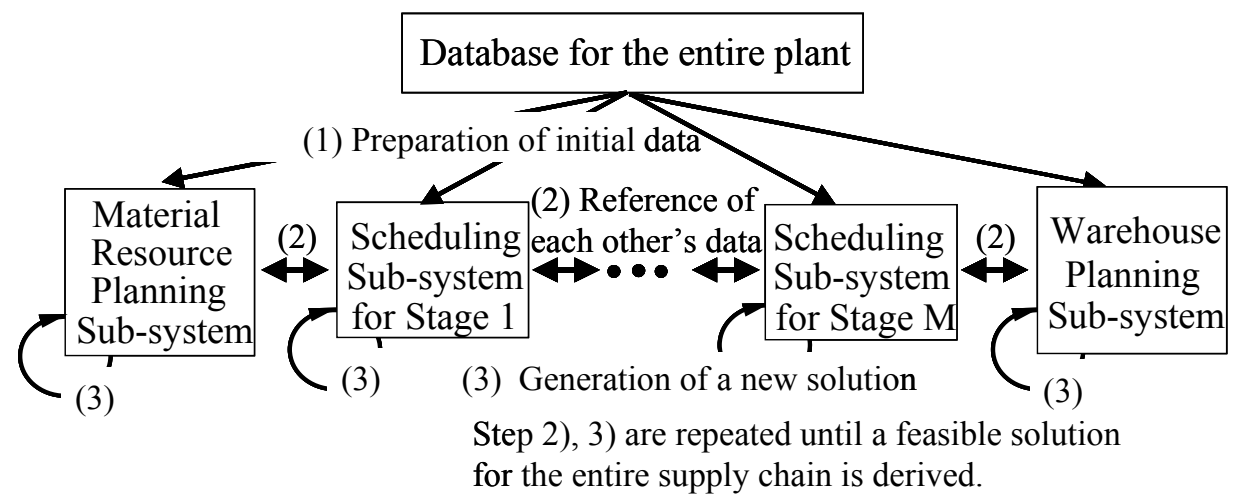

Figure 2. System structure of autonomous decentralized supply chain planning and scheduling system 
Each sub-system has own local decision variables and an objective function. The decision variable and the objective function at each sub-system are also denoted in Figure 3.

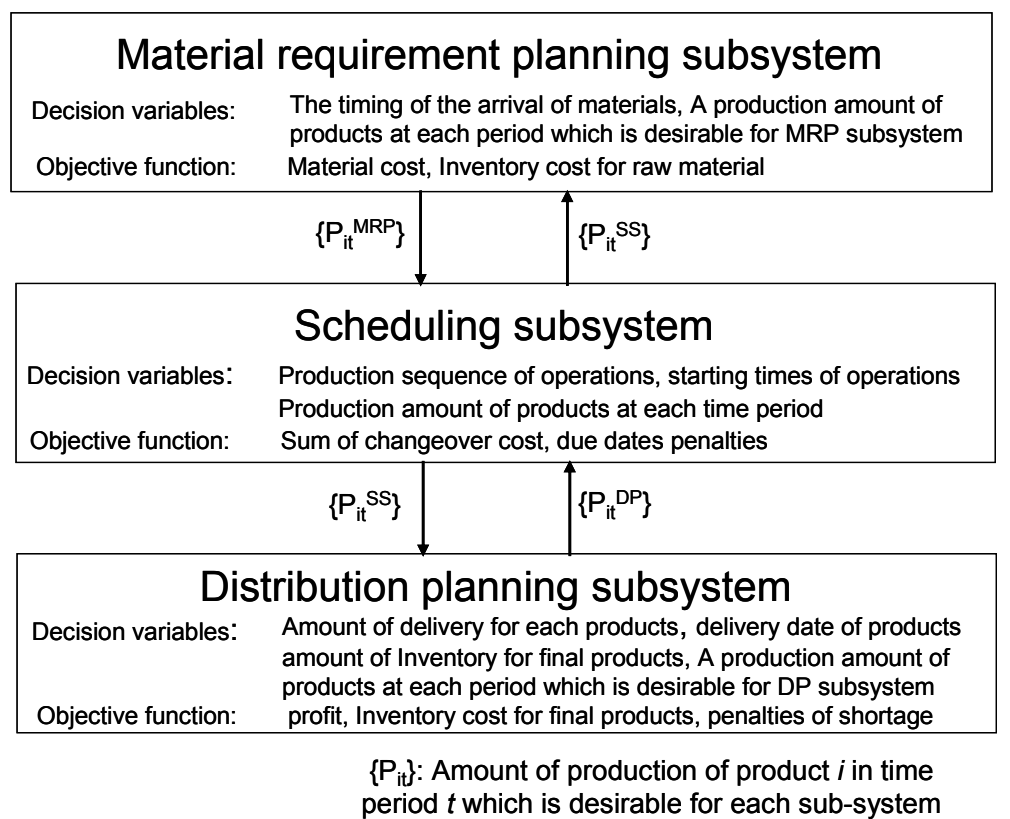

Figure 3. Model structure of the autonomous decentralized supply chain planning and scheduling system

Each subsystem generates a solution of own local optimization problem. However, if the solutions generated at sub-systems are combined, the obtained solutions are infeasible in most cases. To make the solution feasible, each subsystem contacts the other subsystems and exchanges the data among the sub-systems. The data exchanged between the subsystems is the amount of products produced in each time period $P_{i, t}$ derived at each sub-system. The superscripts MRP, SS and DP for $P_{i, t}$ indicate the data generated at the MRP subsystem, the scheduling sub-system, and the DP subsystem respectively. For the proposed system, both of data exchange and re-optimization at each subsystem are repeated several times until a feasible solution for the entire plant is derived. While repeating the data exchange and the re-optimization at each subsystem, penalties for violating the constraints among the subsystems are increased. If the solutions derived at subsystems satisfy feasible conditions for the entire plant, the proposed system generates a total plan and a schedule for the entire plant by combining the solution of all sub-systems. The detail of each subsystem is explained in the following section. 


\subsection{Material requirement planning subsystem}

Material requirement planning subsystem determines the timing and amount of raw material arrived at the production process in each time period. $M_{r, t}$ represents the amount of raw material $r$ arrived at the start of time period $t$, $C_{r, t}$ represents the amount of inventory for raw material $r$ at the end of time period $t$, and $P_{i, t}^{M R P}$ represents the production amount of product $i$ in time period $t$ which is calculated by MRP subsystem. $Y_{r, t}$ denotes the $0-1$ variables indicating whether material $r$ is arriving at the start of time period $t$ or not. Therefore, the optimization problem at the MRP subsystem is formulated as the following mixed integer linear programming problem (MILP).

$$
\begin{gathered}
(\mathrm{MRP}) \min \left(\sum_{r, t} p_{r, t} M_{r, t}+\sum_{r, t} q_{r, t} C_{r, t}+\rho \sum_{i, t} P N_{i, t}\right) \\
C_{r, t}=C_{r, t-1}+M_{r, t}-\sum_{i \in U_{r}} P_{i, t}(\forall r, \forall t) \\
M_{r, t} \leq M_{r, t}^{\max } \cdot Y_{r, t}(\forall r, \forall t) \\
P N_{i, t} \geq\left|P_{i, t}^{M R P}-P_{i, t}^{S S}\right|(\forall i, \forall t) \\
C_{r, t} \leq C_{r, t}^{\max }(\forall r, \forall t) \\
\sum_{t} Y_{r, t} \leq m_{r}(\forall r) Y_{r, t}=\{0,1\}(\forall r, \forall t) \\
M_{r, t}, C_{r, t}, P_{i, t}^{M R P}, P N_{i, t} \geq 0(\forall i, \forall r, \forall t)
\end{gathered}
$$

where,

- $I_{r}$ : set of products produced from material $r$,

- $m_{r}$ : maximum number of the arrivals of raw material $r$ in the total time horizon, 
- $\quad p_{r, t}$ : price of the unit amount of raw material $r$ from supplier to the pro duction process at the start of time period $t$,

- $P_{i, t}^{S S}$ : amount of product $i$ produced in time period $t$, which is obtained from the SS subsystem,

- $P N_{i, t}$ : penalty for infeasibility of the schedule between $P_{i, t}^{M R P}$ and $P_{i, t}^{S S}$,

- $q_{r, t}$ : inventory holding cost of unit amount of raw material $r$ for the dura tion of time period $t$,

- $U_{r}$ : set of products produced from material $r$,

- $\rho$ : weighting factor of the penalty for violating the schedule derived at MRP subsystem and SS subsystem.

\subsection{Scheduling subsystems}

In this section, the scheduling algorithm of the SS subsystem is explained. The flowshop scheduling problem for the SS subsystem is formulated as:

$$
\begin{gathered}
\text { (SS) } \min \left(\sum_{k} C h^{k}+\rho \sum_{i, t}\left(\left|P_{i, t}^{M R P}-P_{i, t}^{S S}\right|+\left|P_{i, t}^{S S}-P_{i, t}^{D P}\right|\right)\right) \\
t_{i}^{k}-t_{j}^{k} \geq s_{j}^{k} \vee t_{j}^{k}-t_{i}^{k} \geq s_{i}^{k} \quad(\forall i, \forall j, \forall k, i \neq j)
\end{gathered}
$$

Where

- $C h^{k}$ is the sequence dependent changeover cost at stage $k$,

- $s_{i}^{k}$ is the processing time of job $i$ at stage $k$.

The second and third terms in Eq. (8) indicate the penalty for the infeasibility of the schedule of SS subsystem with MRP subsystem, and with DP subsystem respectively. Eq. (9) indicates the sequence constraints of operations. The number of jobs for each product is not fixed in advance. Thus, at first, jobs are created by using the production data: $P_{i, t}^{D P}$ obtained from DP subsystem. The number of jobs for each product $i$ is calculated by $\sum P_{i, t}^{D P} / V_{l}$, where $V_{l}$ is the volume of the unit at the production stage $l$. The due date of each product is calculated so that the production amount of each product satisfies its due date. The earliest starting time of each job is calculated by $P_{i, t}^{M R P}$ in the same manner. 
The above procedure makes it possible to adopt the conventional algorithms for solving the scheduling problem. In this paper, the simulated annealing method is used to solve the scheduling problem at each stage.

A scheduling subsystem belonging to each production stage generates a nearoptimal schedule for respective production stage in the following steps:

\section{Preparation of an initial data}

The scheduling subsystem contacts the database for the entire plant and obtains the demand data, such as product name. By using these data, each scheduling subsystem generates the list of jobs to be scheduled. Each job has its earliest starting time and due date. Each job is divided into several operations for each production stage. For each operation, the absolute latest ending time of job $j$ for stage $k$, represented by ALET: $F_{j}^{k}$ is calculated. Here, ALET is the ending time for the stage calculated under the condition that the job arrived at the plant is processed without any waiting time at each stage. ALET means the desired due date for each operation at each production stage.

2. Generation of an initial schedule

Each scheduling subsystem independently generates a schedule of its own production stage without considering the schedules of other stages.

\section{Data exchange among the subsystems}

The scheduling subsystem contacts the DP subsystem and MRP subsystem, and obtains $P_{i, t}^{D P}$ : the production amount of products which is desirable for DP subsystem and $P_{i, t}^{M R P}$ : the production amount of products which is desirable for MRP subsystem. By using these data, each scheduling subsystem modifies the list of jobs to be scheduled. Each job is divided into several operations for each production stage.

The scheduling subsystem belonging to production stage contacts the other scheduling subsystems and exchanges the following data.

a) The tentative earliest starting time (TEST) for each job $j: e_{j}^{k}$ The ending time of job $j$ at the immediately preceding produc tion stage

b) The tentative latest ending time (TLET) for each job $j: f_{j}^{k}$ The starting time of job $j$ at the immediately following produc tion stage 
Figure 4 illustrates the situation of scheduling for SS subsystem of production stage 2 on the condition that the schedules of the production stage 1 and stage 3 are fixed. TEST and TLET of job A at the production stage 2 are shown respectively. It is assumed that every job has the path from stage 1 through stage 3 . TEST of job A at stage 2 indicates the ending time of job $A$ at stage 1, and TLET of job $A$ at stage 2 indicates the starting time of job A at stage 3 . If the starting time of job A at stage 2 is earlier than TEST or the ending time of job $A$ at stage 2 is later than TLET, the schedule is infeasible. Therefore, penalty of violating the feasibility of schedule is embedded in the objective function in the optimization at each scheduling subsystem for respective production stage.

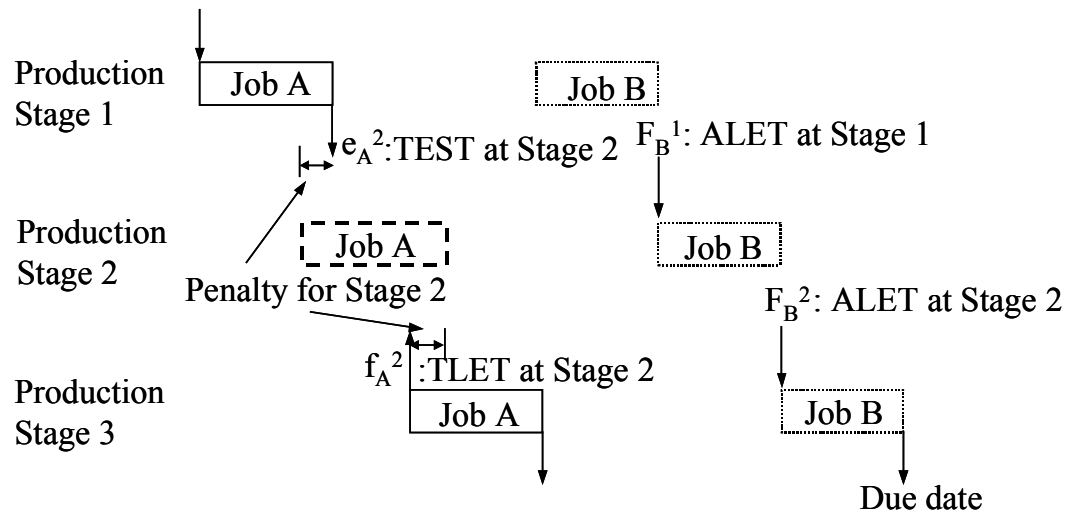

Figure 4. Tentative earliest starting time (TEST), tentative latest ending time (TLET) and absolute latest ending time (ALET)

4. Optimization of the schedule for each SS subsystem

Using the data obtained at step 3), the scheduling subsystem optimizes the production sequence of operations for that production stage. In order to include tardiness penalties in the objective function at every production stage, tardiness from ALET is embedded in the objective function. The scheduling problem for each scheduling subsystem is shown in Eq. (10). The term having weighting factor corresponds to the penalty for violating the precedence constraints with the preceding production stage and the following production stage respectively.

$$
\begin{gathered}
(\mathrm{SS}-k) \min \left[C h^{k}+\sum_{j} \max \left(0, t_{j}^{k}-F_{j}^{k}\right)\right. \\
+\rho\left\{\sum_{j} \max \left(0, e_{j}^{k}-t_{j}^{k}\right)+\sum_{j} \max \left(0, t_{j}^{k}-f_{j}^{k}\right)\right\}
\end{gathered}
$$


s.t. Eq. (9)

where,

$-t_{j}^{k}$ is the starting time of operation for job $j$ at stage $k$.

The optimization problem (SS- $k$ ) for each SS subsystem is solved by using Simulated Annealing (SA) method combined with a neighbourhood search algorithm (Nishi et al., 2000b). The outline of the scheduling algorithm is composed of the following steps.

a) Generate an initial production sequence of operations and calculate the starting times of operations, and calculate the objective function.

b) Select an operation randomly and insert the selected operation into a randomly selected position, thereby change the processing order of operations.

c) For a newly generated production sequence, calculate the starting times of operations by the forward simulation and calculate the objective function. And then decide whether the newly generated schedule is adopted or not by using the criterion of simulated annealing method.

d) Repeat the procedure (b) to (c) for a predetermined number of times $\left(N_{S}\right)$ at the same temperature parameter $\left(T_{S A}\right)$, then the temperature parameter is reduced $T_{S A} \leftarrow \eta T_{S A}$, where $\eta$ is annealing ratio. Then repeat (b) to (d) for a predetermined number of times $\left(N_{A}\right)$.

A production schedule with a minimum objective function is regarded as the current optimal sequence. From the results of production sequence obtained by the simulated annealing method, the starting times of operations are calculated and the production amount of each products in each time period $P_{i, t}^{S S}$ is calculated by using the schedule generated by the simulated annealing method. In the proposed system, any scheduling model and any optimization algorithm can be adopted in the scheduling subsystem. Therefore, the proposed system can easily applicable to many types of scheduling problems such as jobshop problem (Hasebe et al., 1994), flowshop problem with intermediate storage constraints (Nishi et al., 2000c) by changing the algorithm of starting time calculation. 


\subsection{Distribution planning subsystem}

When the lower and upper bound of the amount of production demand for the duration of group-time periods: $S_{i, m}^{\min }, S_{i, m}^{\max }$ are given at each group-time periods (week, or month), the DP subsystem determines the delivery plan to customers so as to maximize the profit taking the inventory cost and the penalties of product shortage. Thus, the optimization problem at the DPsubsystem is formulated as follows:

$$
\begin{gathered}
\text { (DP) } \min \left[\sum_{i, t} h_{i, t} I_{i, t}+\sum_{i, t} v_{i, t} P_{i, t}^{D P}+\sum_{i, m} \varsigma_{i, m} I_{i, m}^{-}\right. \\
\left.-\sum_{i, t} \mu_{i, t} S_{i, t}+\rho \sum_{i, t} P N_{i, t}\right] \\
I_{i, t}=I_{i, t-1}+P_{i, t}^{D P}-S_{i, t} \quad(\forall i, \forall t) \\
I_{i, m}^{-} \geq S_{i, m}^{\min }-\sum_{t^{\prime} \in T_{k}} S_{i, t^{\prime}} \quad(\forall i, \forall m) \\
P N_{i, t} \geq\left|P_{i, t}^{D P}-P_{i, t}^{S S}\right| \quad(\forall i, \forall t) \\
I_{i, t} \leq I_{i, t}^{\max } \quad(\forall i, \forall t) \\
\sum_{t \in T_{m}} S_{i, t} \leq S_{i, m}^{\max }(\forall i, \forall m) \\
I_{i, t}, I_{i, m}^{-}, P_{i, t}^{D P}, S_{i, t}, P N_{i, t} \geq 0 \quad(\forall i, \forall m, \forall t)
\end{gathered}
$$

where,

- $\mu_{i, t}$ : revenue of product $i$ sold in time period $t$,

- $h_{i, t}$ : inventory cost for holding unit amount of final product $i$ for the duration of time period $t$,

- $I_{i, t}$ : inventory level of final product $i$ at the end of time period $t$,

- $I_{i, m}^{-}$: amount of shortage of final product $i$ in group-time periods $m$,

- $S_{i, t}$ : amount of final product $i$ delivered in time period $t$,

- $T_{m}$ : set of group-time periods $m$,

- $v_{i, t}$ : production cost of product $i$ in time period $t$. 
Eq. (11) is the objective function of DP subsystem which is the sum of the inventory holding cost for final products, production costs, penalty for product shortage, revenue of products and penalty for violating the constraints with SS subsystem. Eq. (12) indicates a material balance equation around the storage space for final product. Eq. (13) indicates the constraints on the minimum demand. Eq. (14) indicates the penalty value for violating the constraints imposed by the scheduling subsystem. Eq. (15) shows the capacity constraints of holding the final products in the storage space. Eq. (16) denotes the constraint of maximum amount of delivery to customer. Eq. (17) indicates the non-negative value constraints of all the decision variables.

\subsection{Overall optimization algorithm}

The total subsystem derives a feasible schedule by the following steps.

Step 1. Preparation of the initial data.

Each subsystem contacts the database and obtains the data and initializes the weighting factor of the penalty term, e.g. $\rho \leftarrow 0$.

Step 2. Generation of an initial solution.

Each subsystem independently generates a solution without considering the other subsystems.

Step 3. Exchanging the data.

Each subsystem contacts the other sub-systems and exchanges the amount of product data: $P_{i, t}^{M R P}, P_{i, t}^{S S}, P_{i, t}^{D P}$.

Step 4. Judging whether the optimization at each subsystem is skipped or not.

To avoid cyclic generation of same solutions, each subsystem skips Step 5 with a predetermined probability (see Hasebe et al., 1994).

Step 5. Optimization at each subsystem.

By using the data obtained at step 3, each subsystem executes the optimization of each subproblem.

Step 6. Judging the convergence.

When the solutions of all subsystems satisfy both of the following conditions, all of the subsystems stop the calculation, and the derived solution is regarded as the final solution.

The solution generated at Step 5 is the same as that generated at Step 5 in the previous iteration. 
The value of the penalty function embedded in the objective function is equal to zero.

Step 7. Updating the weighting factor.

If the value of penalty function is positive, the derived solution is infeasible. Therefore, in order to reduce the degree of infeasibility, the weighting factor of the penalty term is increased. The value of weighting factor for penalty is updated by $\rho \leftarrow \rho+\Delta \rho$ at each iteration. Then return to Step 3. The incremental value $\Delta \rho$ is a constant. If the value of $\Delta \rho$ is larger, the performance index of solution derived by the proposed system becomes worse, on the other hand, the total computation time becomes shorter. On the contrary, if the value of $\Delta \rho$ is smaller, the total computation time is increased while the value of performance index has been improved. Our numerical studies show that when $\Delta \rho$ is less than 0.5, the computation time becomes exponentially larger even though the performance is not so improved. From these results, we have determined $\Delta \rho=0.5$ as shown in Table 8 .

By taking the above algorithm, it is easy for the proposed system to introduce the parallel processing system using multiple computers in which each subsystem execute its optimization concurrently. Figure 5 is a diagram showing the data exchange algorithm of the proposed system. Each square in Figure 5 illustrates steps of the data exchange algorithm in the iteration of the optimization at the subsystem and each arrow represents the flow of data. The total number of the processors required for solving the supply chain optimization problem is 5 processors for 3-stage production processes. The dotted arrow indicates the data of the production amount of each product in each time period determined by the MRP subsystem and DP subsystem. The thick arrow indicates the tentative earliest starting time (TEST) and tentative latest starting time (TLET) which are exchanged among the SS subsystems. In each iteration step, the data of the amount of products in each time period calculated in each subsystem are transmitted. Then, the data of TEST and TLST are exchanged. Therefore, jobs are generated at each iteration in the proposed system. While repeating the data exchange among each subsystem, the number of jobs and the starting time of operations are gradually satisfied with the constraints among each subsystem. 


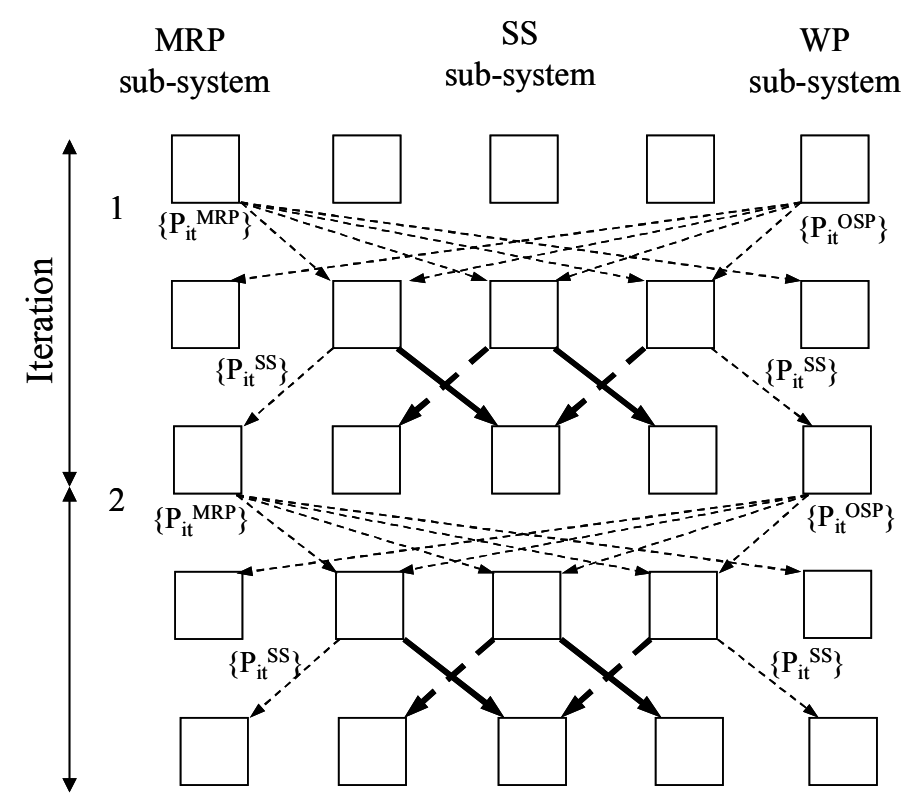

Figure 5. Data exchange algorithm

\section{Computational results}

The proposed scheduling system is applied to a supply chain optimization problem. The MRP subsystem and the DP subsystem is solved by a commercial MILP solver (CPLEX8.0 iLOGC). The algorithm used in the scheduling subsystem is coded by $\mathrm{C}++$ language. Pentium IV $(2.0 \mathrm{AGHz})$ processor is used for computation.

\subsection{Example problem}

A batch plant treated in the example problem consists of three production stages shown in Figure 6. In this example, it is assumed that the production paths of all jobs are the same, meaning the each job is processed at stages 1 through 3. In this plant, four kinds of products are produced by each of two kinds of raw materials. Product A or B is produced from material 1, and product $\mathrm{C}$ and $\mathrm{D}$ is produced from material 2. The total planning horizon is 12 days, and it is divided into 12 time periods in the MRP subsystem and in the DP subsystem. The shipping of raw material for each material is available only two times in 4 days $(1,4,7,10)$. For each product, the lower bound and the upper bound of the production demand for each 4 days are given as the ag- 
gregated value for each product. Thus, the delivery date can be decided by the DP subsystem. The plant is operated 24 hours/day. The available space for inventory for raw material and final products are restricted. The tables 1 to 8 show the data and parameters used in the example problem.

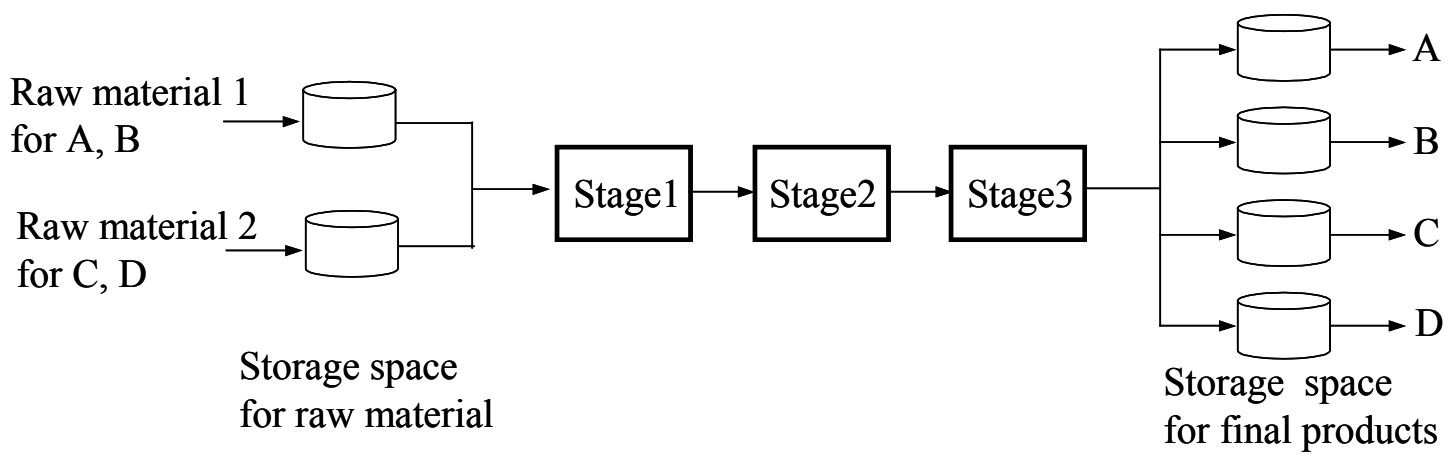

Figure 6. 3-stage production process for the example problem

\begin{tabular}{lllllllllllll}
\hline$J / T$ & 1 & 2 & 3 & 4 & 5 & 6 & 7 & 8 & 9 & 10 & 11 & 12 \\
\hline $\mathrm{A}$ & 1.90 & 2.00 & 1.80 & 1.95 & 2.00 & 1.95 & 2.10 & 2.00 & 1.80 & 1.90 & 2.20 & 2.30 \\
$\mathrm{~B}$ & 2.20 & 2.30 & 2.15 & 2.15 & 2.25 & 2.30 & 2.15 & 2.20 & 2.35 & 2.40 & 2.25 & 2.50 \\
$\mathrm{C}$ & 2.00 & 2.20 & 2.20 & 3.20 & 3.25 & 2.25 & 1.10 & 1.25 & 2.05 & 2.10 & 1.35 & 2.30 \\
$\mathrm{D}$ & 2.20 & 2.50 & 2.20 & 2.50 & 1.30 & 2.20 & 3.50 & 1.20 & 2.20 & 3.70 & 2.60 & 2.40 \\
\hline
\end{tabular}

Table 1. Revenue of products at each time period

\begin{tabular}{llll}
\hline$J$ & $T: 1-4$ & $T: 5-8$ & $T: 9-12$ \\
\hline A & 0.45 & 0.45 & 0.45 \\
B & 0.45 & 0.45 & 0.45 \\
C & 0.60 & 0.60 & 0.60 \\
D & 0.60 & 0.60 & 0.60 \\
\hline
\end{tabular}

Table 2. Production costs

\begin{tabular}{llll}
\hline$J$ & $T: 1-4$ & $T: 5-8$ & $T: 9-12$ \\
\hline A & 100 & 200 & 100 \\
B & 100 & 200 & 400 \\
C & 200 & 300 & 300 \\
D & 100 & 100 & 400 \\
\hline
\end{tabular}

Table 3. Minimum demand data 


\begin{tabular}{llll}
\hline$J$ & $T: 1-4$ & $T: 5-8$ & $T: 9-12$ \\
\hline A & 1100 & 900 & 1200 \\
B & 1200 & 1400 & 1100 \\
C & 2200 & 2500 & 2000 \\
D & 1000 & 800 & 1000 \\
\hline
\end{tabular}

Table 4. Maximum demand data

\begin{tabular}{llllll}
\hline Stage & From/to & A & B & C & D \\
\hline 1 & A & 0 & 10 & 10 & 10 \\
& B & 40 & 0 & 10 & 10 \\
& C & 40 & 40 & 0 & 10 \\
& D & 40 & 40 & 40 & 40 \\
\hline 2 & A & 0 & 0 & 30 & 30 \\
& B & 0 & 0 & 30 & 30 \\
& C & 30 & 30 & 0 & 0 \\
& D & 30 & 30 & 0 & 0 \\
\hline 3 & A & 0 & 30 & 20 & 10 \\
& B & 10 & 0 & 30 & 20 \\
& C & 20 & 10 & 0 & 30 \\
& D & 30 & 20 & 10 & 0 \\
\hline
\end{tabular}

Table 5. Sequence dependent changeover cost data

\begin{tabular}{lllllllllllll}
\hline$J / T$ & 1 & 2 & 3 & 4 & 5 & 6 & 7 & 8 & 9 & 10 & 11 & 12 \\
\hline $\mathrm{A}$ & 1.90 & 2.00 & 1.80 & 1.95 & 2.00 & 1.95 & 2.10 & 2.00 & 1.80 & 1.90 & 2.20 & 2.30 \\
$\mathrm{~B}$ & 2.20 & 2.30 & 2.15 & 2.15 & 2.25 & 2.30 & 2.15 & 2.20 & 2.35 & 2.40 & 2.25 & 2.50 \\
$\mathrm{C}$ & 2.00 & 2.20 & 2.20 & 3.20 & 3.25 & 2.25 & 1.10 & 1.25 & 2.05 & 2.10 & 1.35 & 2.30 \\
$\mathrm{D}$ & 2.20 & 2.50 & 2.20 & 2.50 & 1.30 & 2.20 & 3.50 & 1.20 & 2.20 & 3.70 & 2.60 & 2.40 \\
\hline
\end{tabular}

Table 6. Price and data of raw material

\begin{tabular}{lllll}
\hline & $A$ & $B$ & $C$ & $D$ \\
\hline Stage 1 & 4 & 5 & 2 & 5 \\
Stage 2 & 5 & 5 & 2 & 4 \\
Stage 3 & 3 & 5 & 4 & 4 \\
\hline
\end{tabular}

Table 7. Processing time data 


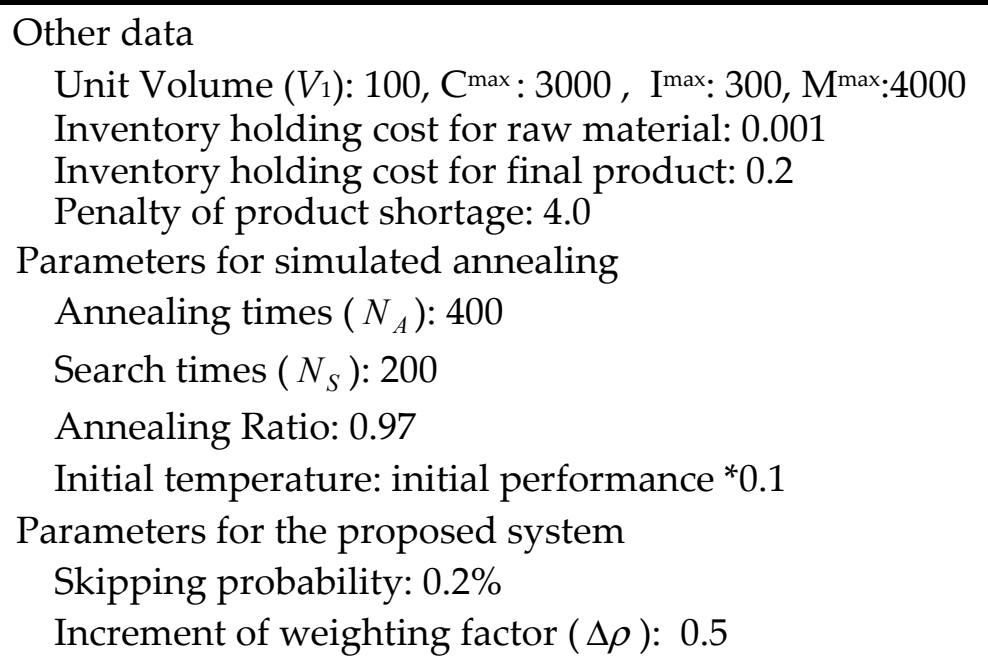

Table 8.Parameters used for computation

\subsection{Results of coordination}

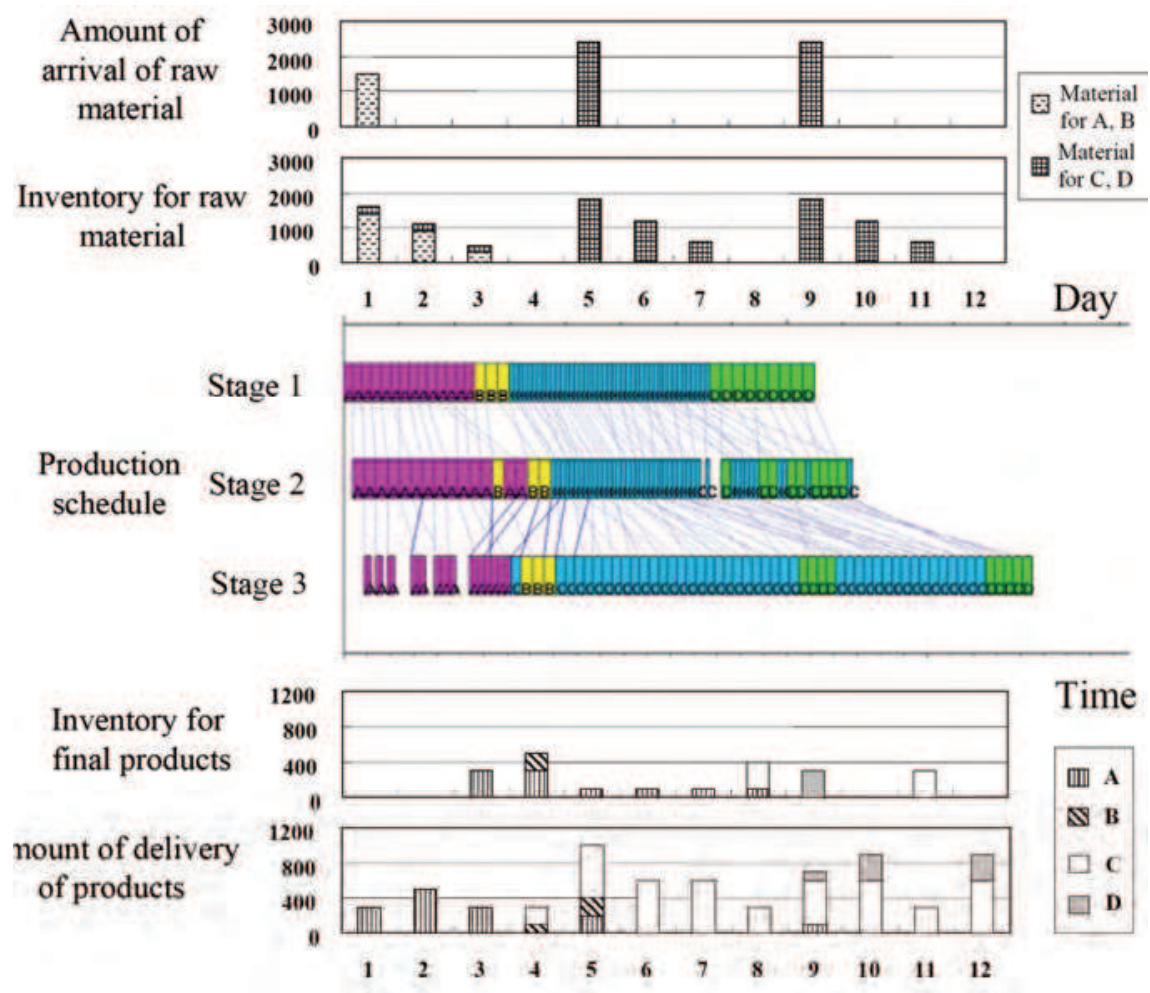

Figure 7. Intermediate result after 10 times of data exchange 
Figure 7 shows an intermediate result obtained after ten times data exchange. This result is infeasible because several jobs at stage 3 are finished later than the scheduling horizon (Day 12). This is because the DP subsystem individually tries to generate a distribution planning so that the amount of delivery amount to the customer is maximized. Therefore, the planning result is also infeasible because the amount of production at stage 3 in each time period is not equivalent to the amount of the product delivery. Moreover, several operations at stage 2 are finished later than the starting times of operations at stage 3 .

Figure 8 shows the final schedule obtained after 23 times of data exchanges. A feasible schedule is obtained by the proposed system. The transitions of the performance index in 23 times of iterations are shown in Figure 9.

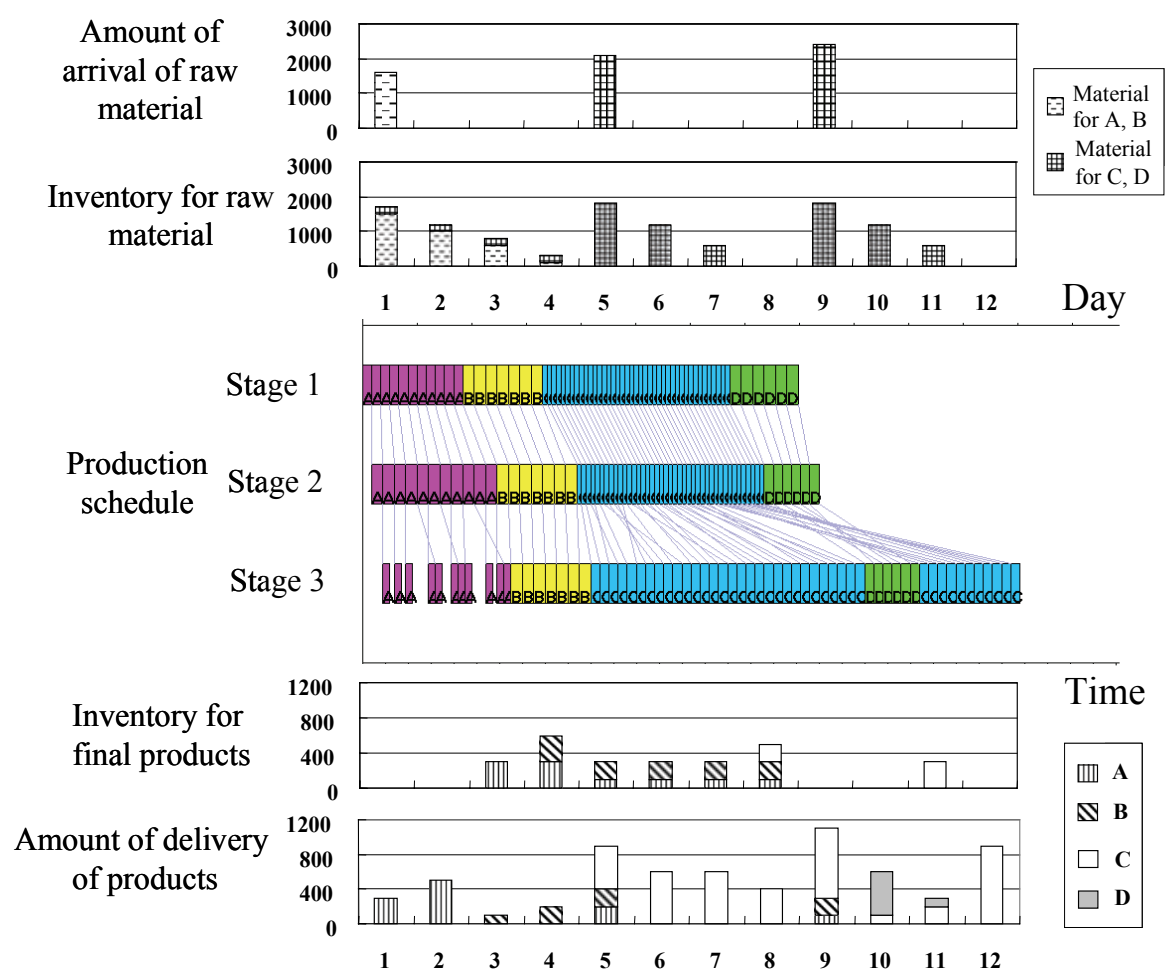

Figure 8. Final result after 23 times of data exchange

As the number of iteration increases, the value of penalty function decreases and it becomes close to zero. This indicates that the proposed system gradually generates a feasible solution by increasing the value of weighting factor of the penalty for violating the material balancing constraints although only the local information is used to optimize the objective function for each subsystem. 


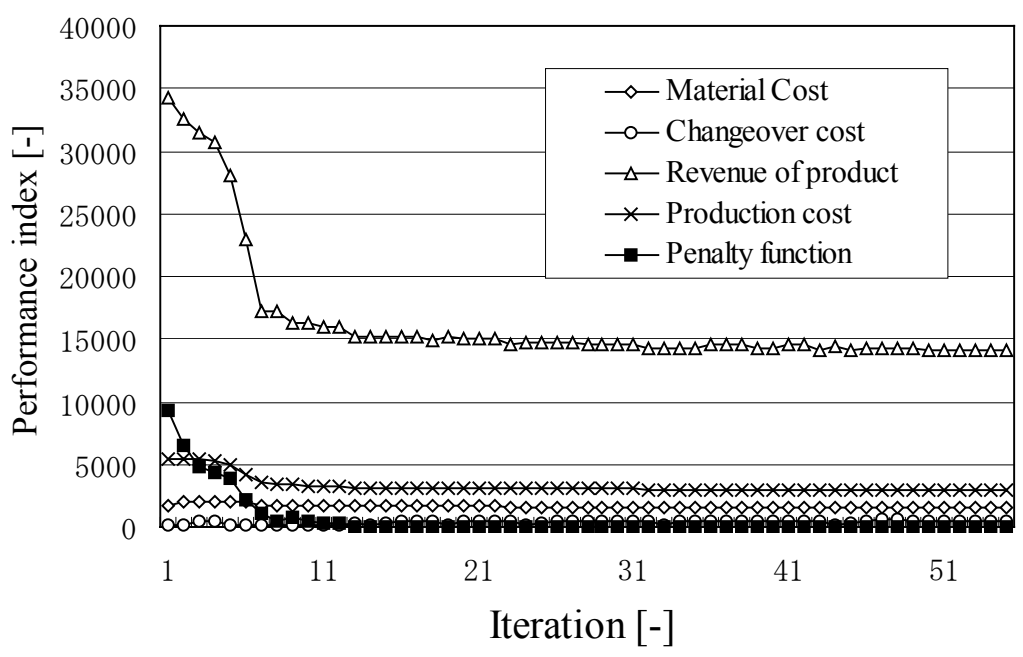

Figure 9. The transitions of the performance index

\subsection{Comparison of the proposed system and the conventional system}

In order to evaluate the performance of the proposed system, a hierarchical planning and scheduling system considering the entire plant is also developed. The planning problem (HP) is formulated as MILP problem by using the midterm-planning model proposed by McDonald \& Karimi (1997).

$$
\begin{gathered}
(\mathrm{HP}) \min Z Z=\sum_{r, t} p_{r, t} M_{r, t}+\sum_{r, t} q_{r, t} C_{r, t}+\sum_{i, t} v_{i, t} P_{i, t} \\
+\sum_{i, t} h_{i, t} I_{i, t}+\sum_{i, m} S_{i, m} I_{i, m}^{-}-\sum_{i, t} \mu_{i, t} S_{i, t} \\
C_{r, t}=C_{r, t-1}+M_{r, t}-\sum_{i \in U_{r}} P_{i, t}(\forall r, \forall t) \\
C_{i, t} \leq C_{i, t}^{\max }(\forall i, \forall t) \\
\sum_{t} Y_{r, t} \leq m_{r}(\forall r) \\
\sum_{i} s_{i}^{l} \cdot P_{i, t} / V_{l} \leq H_{t}(\forall t, \forall l)
\end{gathered}
$$




$$
\begin{gathered}
I_{i, t}=I_{i, t-1}+P_{i, t}-S_{i, t}(\forall i, \forall t) \\
I_{i, t} \leq I_{i, t}^{\max }(\forall i, \forall t) \\
I_{i, m}^{-} \geq S_{i, m}^{\min }-\sum_{t^{\prime} \in T_{m}} S_{i, t^{\prime}}(\forall i, \forall m) \\
P_{i, t}, M_{i, t}, C_{i, t}, I_{i, t}, I_{i, t}^{-}, S_{i, t} \geq 0(\forall i, \forall t) \\
Y_{i, t} \in\{0,1\} \quad(\forall i, \forall t)
\end{gathered}
$$

where,

- $H_{t}$ : amount of time available in the time period $t$,

- $\quad T_{m}$ : set of group-time period $m$,

- $\quad V_{t}$ : batch size of the machine at the production stage $l$.

For the hierarchical approach, the solution of the HP problem is transferred to the scheduling subsystem as the production request. The jobs are created by using the production amount: $P_{i, t}$ calculated in the planning system. The scheduling system obtains the amount of inventory for raw material, due dates for each job. And then the scheduling system is executed. For the scheduling system used in the hierarchical approach, a schedule considering the entire production stages is successively generated improving an initial schedule. The simulated annealing method is adopted so that the solution is not trapped in a local optimal.

Ten times of calculations are made with different seed numbers for generating random numbers in the simulated annealing method to compare the performance of the proposed system. The results of the performance index for the proposed system (DSCM1) and the hierarchical planning and scheduling system (CONV) are shown in Table 10. The average computation time for deriving a feasible schedule of the proposed algorithm is 198 seconds. The performance of the DSCM1 is lower than that of the hierarchical system (CONV). This is because some of the solutions of the schedule generated by each subsystem have been entirely different from that derived by other subsystem, which makes convergence of the proposed algorithm difficult. Thereby, the final solution of the proposed system has been trapped into a bad local optimum. In order to improve the efficiency of the proposed method, the capacity constraints for 
each month are embedded into the DP subproblem. By adding the capacity constraints to DP subproblem, the number of generating meaningless solutions violating the constraints with the SS subsystem has been reduced. The results of computation for the improved method (DSCM2) are shown in Table 10. The profit of DSCM2 is successfully improved compared with that of DSCM1 without sacrificing the computational expenses. For DSCM2, the changeover cost is lower than that of CONV, though the profit for final products is higher than CONV. This is because both the changeover costs and the profit of the product greatly depend on the production schedule, and it is very difficult for CONV to determine the precise production schedule at the production planning level. It is demonstrated that the total profit of proposed system (DSCM2) is higher than that of the conventional system (CONV). The proposed system can generate a better solution than the conventional system even though only local information is used to generate the solution of each subsystem.

\section{Conclusion and future work}

An autonomous decentralized supply chain optimization system for multistage production processes has been proposed. The novel aspect of this paper is that we provide a novel distributed optimization system for a supply chain planning problem for multi-stage production processes comprising a material requirement planning (MRP) subsystem, scheduling subsystems for each production stage, and a distribution planning (DP) subsystem.

\begin{tabular}{llll}
\hline Methods & DSCM1 & DSCM2 & CONV \\
\hline Number of data exchange & 18 & 21 & 1 \\
Profit [-] & 7,533 & 9,538 & 9,418 \\
Number of jobs [-] & 66 & 66 & 62 \\
Total revenue [-] & 14,353 & 15,759 & 15,460 \\
Costs of raw material [-] & 1,540 & 1,688 & 1,677 \\
Inventory cost for raw materials [-] & 18 & 21 & 26 \\
Inventory holding cost for final products [-] & 504 & 720 & 360 \\
Production cost [-] & 2,970 & 3,006 & 2,790 \\
Penalty of product shortage [-] & 1320 & 360 & 400 \\
Sequence dependent changeover cost [-] & 468 & 426 & 1,030 \\
\hline
\end{tabular}

Table 10. Comparison of the autonomous decentralized supply chain planning system (DSCM) and the conventional system (CONV) 
Each subsystem includes an optimization function and repeats the generation of solutions for each subproblem and data exchange among the subsystems. The total system derives a feasible solution by gradually increasing the weighting factor for violating the infeasibility of the solution through repeated optimization at each subsystem and data exchanges among the subsystems. The data exchanged among the subsystems are tentative production amount of each product at each time period that is desirable for each subsystem. By adopting such a structure, it is easy to modify the subsystem when a new constraint is added or when performance evaluation criteria changes. Thus, the system can flexibly accommodate various unforeseen changes. he proposed system is successfully applied to a multi-stage supply chain optimization problem. The results demonstrate that feasible solutions could be obtained by the numerical examples. The performances of the proposed system are compared with those of the schedule derived by the conventional system. It has been shown that the proposed system can generate a better solution than the conventional system without sacrificing flexibility and computational resources. Future work should be investigated on how to optimize the entire supply chain under several uncertainties.

\section{Nomenclature}

- $C_{r, t}$ : amount of inventory of raw material $r$ at the end of time period $t$,

- $C_{r, t}^{\max }$ : maximum amount of inventory for raw material $r$ at the end of time period $t$,

- $C h^{k}$ : sequence dependent changeover cost at stage $k$,

- $e_{j}^{k}$ : tentative earliest starting time of job $i$ at stage $k$,

- $f_{j}^{k}$ : tentative latest ending time of job $i$ at stage $k$,

- $F_{j}^{k}$ : absolute latest ending time of job $j$ at stage $k$,

- $h_{i, t}$ : inventory cost for holding unit amount of final product $i$ for the duration of time period $t$,

- $I_{i, t}$ : inventory level of final product $i$ at the end of time period $t$,

- $\quad I_{i, t}^{\max }$ : maximum amount of inventory for final product $i$ at the end of time period $t$,

- $I_{i, m}^{-}$: amount of shortage of inventory for final product $i$ in group-time periods $m$,

- $K$ : sufficiently large positive number, 
- $m_{r}:$ maximum number of the arrival of raw material $r$,

- $M_{r, t}$ : amount of raw material $r$ arrived from supplier at the start of time period $t$,

- $N_{A}$ : annealing time for simulated annealing,

- $N_{S}$ : search times at the same temperature for simulated annealing,

- $p_{r, t}$ : price of the unit amount of raw material $r$ from supplier to the plant at the start of time period $t$,

- $P_{i, t}^{M R P}$ tentative amount of production of product $i$ in time period $t$, which is derived at MRP subsystem,

- $\quad P_{i, t}^{D P}$ : tentative amount of production of product $i$ in time period $t$, which is derived at DP subsystem,

- $P_{i, t}^{S S}$ : tentative amount of production of product $i$ in time period $t$, which is derived at DP subsystem,

- $P N_{i, t}$ : difference of production amount of product $i$ in time period $t$,

- $q_{r, t}$ : inventory holding cost of unit amount of raw material $r$ for the duration of time period $t$,

- $s_{i}^{k}$ : processing time of operation for job $j$ at stage $k$,

- $S_{i, t}$ : amount of final product $i$ delivered in time period $t$,

- $t_{j}^{k}$ : starting time of operation for job $j$ at stage $k$,

- $T_{m}$ : set of time in group-time periods $m$,

- $T_{\text {avaibble }}$ periods of time when the material arrival is available,

- $T_{S A}$ : annealing temperature for simulated annealing method,

- TEST tentative earliest starting time,

- TLET tentative latest ending time, $U_{r}$ : set of products produced from material $r$,

- $V_{l}$ : batch size of the machine at the production stage $l$,

- $Y_{r, t}$ : binary variable indicating whether material $r$ is arrived at the start of time period $t$ or not.

\section{Greek Letters}

- $\quad \eta$ : annealing ratio ( temperature reduction factor),

- $\mu_{i, t}$ : revenue of of product $i$ sold in time period $t$,

- $v_{i, t}$ : production cost of product $i$ in time period $t$,

- $\rho$ : penalty parameter,

- $\varsigma_{i, m}$ : penalty for unit amount of shortage of product in group-time periods $m$, 


\section{References}

Androulakis, I. and Reklaitis, G. (1999), Approaches to Asynchronous Decentralized Decision Making, Computers and Chemical Engineering, Vol. 23, pp. 341-355.

Birewar, D. and Grossmann, I. (1990) Production Planning and Scheduling in Multiproduct Batch Plants, Ind. Eng. Chem. Res., Vol. 29, 570-580.

Bitran, R., Hax, A. (1977) On the Design of Hierarchical Production Planning Systems, Decision Sciences, Vol. 8, pp. 29-55.

Bok, J.K., Grossmann, I.E., Park, S. (2000) Supply Chain Optimization in Continuous Flexible Process, Ind. Eng. Chem. Res., Vol. 39, pp. 1279-1290.

Burke, P., Prosser, P., (1990) Distributed Asynchronous Scheduling, Applications of Artificial Intelligence in Engineering V, Vol. 2, pp. 503-522.

Gou, L., Luh, P.B. Luh, Kyoya, Y. (1998) Holonic manufacturing scheduling: architecture, cooperation mechanism, and implementation, Computers in Industry, Vol. 37, pp. 213-231.

Gupta, A., Maranas, C.D. (1999) Hierarchical Lagrangean Relaxation Procedure for Solving Midterm Planning Problems, Ind. Eng. Chem. Res., Vol. 38, pp. 1937-1947

Hasebe, S., Kitajima, T., Shiren, T., Murakami, Y. (1994) Autonomous Decentralized Scheduling System for Single Production Line Processes, Presented at AIChE Annual Meeting, Paper 235c, USA.

Hoitomt, D.J., Luh, P.B., Pattipati, R. (1993) A Practical Approach to Job-Shop Scheduling Problems, IEEE Trans. Robot. Automat., Vol. 9, pp. 1-13.

Jeremy, F. S. (2001) Modeling the Supply Chain, Thomson Learning.

Fischer, K. (1999) Agent-based design of holonic manufacturing systems, Robotics and Autonomous Systems, Vol. 27, pp. 3-13.

Maturana, F.P., Norrie, D.H., (1997) Distributed decision-making using the contract net within a mediator architecture, Decision Support Systems, Vol. 20, pp. 53-64.

McDonald, C. and Karimi, I. (1997), Planning and Scheduling of Parallel Semicontinuous Processes. 1. Production Planning, Ind. Eng. Chem. Res., Vol. 36, pp. 2691-2700.

Mckay, A., Pennington, D., Barnes, C. (2001) A Web-based tool and a heuristic method for cooperation of manufacturing supply chain decisions, Vol. 12, pp. 433-453. 
Nishi, T., Inoue, T., Yutaka, H., Taniguchi, S. (2000a) Development of a Decentralized Supply Chain Optimization System, Proceedings of the International Symposium on PSE Asia, 141-146.

Nishi, T., Konishi, M., Hattori, Y., Hasebe, S. (2003) A Decentralized Supply Chain Optimization Method for Single Stage Production Systems, Transactions of the Institute of Systems, Control and Information Engineers, 16-12, 628-636 (in Japanese)

Nishi, T., Sakata, A., Hasebe, S., Hashimoto, I. (2000b), Autonomous Decentralized Scheduling System for Just-in-Time Production, Computers and Chemical Engineering, Vol. 24, pp. 345-351.

Nishi, T., Sakata, A., Hasebe, S., Hashimoto, I. (2000c), Autonomous Decentralized Scheduling System for flowshop problems with storage cost and due-date penalties, Kagaku Kogaku Ronbunshu, Vol. 26, pp. 661-668 (in Japanese).

Ohkuma, K., Ueda, K., (1996) Solving Production Scheduling Problems with a Simple Model of Biological-Oriented Manufacturing Systems, Nihon Kikaigakkai Ronbunshu C, Vol. 62, pp. 429-435, 1996 (in Japanese).

Rupp, T.M., Ristic, M. (2000) Fine Planning for Supply Chains in Semiconductor Manufacture, Journal of Material Processing Technology, Vol. 107, pp. 390-397.

Shaw, M.J., (1987) A distributed scheduling method for computer integrated manufacturing: the use of local area network in cellular systems, Int. J. Prod. Res., Vol. 25, No. 9, pp. 1285-1303.

Simon, C., Pietro, R., Mihalis G. (2000) Supply chain management: an analytical framework for critical literature review, European Journal of Purchasing $\mathcal{E}$ Supply Management, Vol. 6, pp. 67-83.

Smith, R.G., (1980) The Contract Net Protocol: High-Level Communication Control in a Distributed Problem Solver, IEEE Transactions on Computers, Vol. 29, No.12, pp. 1104-1113.

Tan, M., (2001) Hierarchical Operations and Supply Chain Planning, Springer.

Tharumarajah, A., Bemelman, R., (1997) Approaches and issues in scheduling a distributed shop-floor environment, Computers in Industry, Vol. 34, pp. 95-109.

Vidal, C.J. and Goetschalckx, M. (1997) Strategic production-distribution models: A critical review with emphasis on global supply chain models, European Journal of Operational Research, Vol. 98, pp. 1-18.

Wei, T., (2000) Integration of Process Planning and Scheduling - a Review, Journal of Intelligent Manufacturing, Vol. 11, pp. 51-63. 


\section{Manufacturing}

the Future

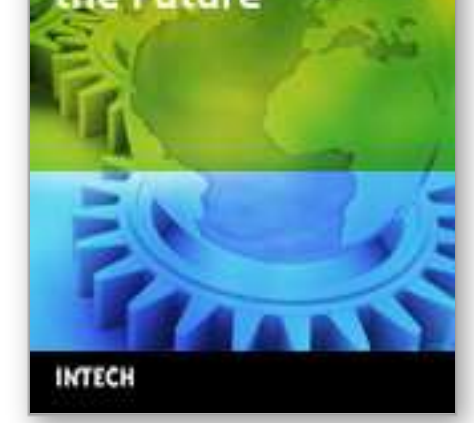

\section{Manufacturing the Future}

Edited by Vedran Kordic, Aleksandar Lazinica and Munir Merdan

ISBN 3-86611-198-3

Hard cover, 908 pages

Publisher Pro Literatur Verlag, Germany / ARS, Austria

Published online 01, July, 2006

Published in print edition July, 2006

The primary goal of this book is to cover the state-of-the-art development and future directions in modern manufacturing systems. This interdisciplinary and comprehensive volume, consisting of 30 chapters, covers a survey of trends in distributed manufacturing, modern manufacturing equipment, product design process, rapid prototyping, quality assurance, from technological and organisational point of view and aspects of supply chain management.

\section{How to reference}

In order to correctly reference this scholarly work, feel free to copy and paste the following:

Tatsushi Nishi (2006). An Autonomous Decentralized Supply Chain Planning and Scheduling System, Manufacturing the Future, Vedran Kordic, Aleksandar Lazinica and Munir Merdan (Ed.), ISBN: 3-86611-198-3, InTech, Available from:

http://www.intechopen.com/books/manufacturing_the_future/an_autonomous_decentralized_supply_chain_pla nning_and_scheduling_system

\section{INTECH}

open science | open minds

\section{InTech Europe}

University Campus STeP Ri

Slavka Krautzeka 83/A

51000 Rijeka, Croatia

Phone: +385 (51) 770447

Fax: +385 (51) 686166

www.intechopen.com

\section{InTech China}

Unit 405, Office Block, Hotel Equatorial Shanghai No.65, Yan An Road (West), Shanghai, 200040, China 中国上海市延安西路65号上海国际贵都大饭店办公楼 405 单元 Phone: +86-21-62489820

Fax: $+86-21-62489821$ 
(C) 2006 The Author(s). Licensee IntechOpen. This chapter is distributed under the terms of the Creative Commons Attribution-NonCommercial-ShareAlike-3.0 License, which permits use, distribution and reproduction for non-commercial purposes, provided the original is properly cited and derivative works building on this content are distributed under the same license. 